\title{
Calidad de vida de pacientes con depresión leve o moderada en México
}

\author{
Quality of life in patients with mild or moderate depression \\ in Mexico
}

*Karina Ramírez, **José Valdez-Torres, ${ }^{* * *}$ Sergio Paredes-Osuna, ${ }^{* * * *}$ Cuitláhuac Valdez-Lafarga, \& ${ }^{* * * *}$ José Aldo Hernández Murúa

\begin{abstract}
Ramírez, K., Valdez-Torres, J. C., Paredes-Osuna, S., Valdez-Lafarga, C., \& Hernández, J. A. (2020). Calidad de vida de pacientes con depresión leve o moderada en México. Revista Ciencias de la Actividad Física UCM, № 21(1), enero-junio, 1-15. DOI: http://doi.org/10.29035/rcaf.21.1.7
\end{abstract}

\begin{abstract}
RESUMEN
Objetivo. Identificar las características generales por género y grado de sobrepeso y obesidad y su afectación sobre la calidad de vida de pacientes con leve y moderada depresión en Sinaloa México. Material y métodos. Se realizó un estudio de corte transversal con 182 pacientes con diagnósticos depresivos de leve a moderado. La muestra se caracterizó por género y grado de sobrepeso y obesidad, y se determinó su impacto y asociación sobre la calidad de vida. La calidad de vida se determinó con la encuesta SF-36. Resultados. Se encontraron diferencias significativas entre los pacientes con depresión leve y moderada ( $p=0.000$ ). El rol físico se vio afectado significativamente por el género ( $p=0.047)$, mientas que la función física $(p=0.016)$ y dolor $(p=0.040)$ por el grado de sobrepeso y obesidad en pacientes con depresión leve. Además, se encontraron correlaciones significativas entre la función física y el dolor con el rol físico, salud general y vitalidad. Conclusiones. La depresión leve afectó las dimensiones de la calidad de vida de función física, dolor y rol físico. La depresión y las dimensiones de función física y dolor se ven afectadas por el grado de sobrepeso y obesidad en los pacientes con leve depresión.
\end{abstract}

Palabras clave

Depresión, sobrepeso y obesidad, calidad de vida.

\footnotetext{
* Doctora en Ciencias (PhD). División de Estudios de Posgrado e Investigación, Instituto Tecnológico de Culiacán. Culiacán, México.

** Doctor en Ciencias (PhD). Centro de Investigación en Alimentación y Desarrollo A.C. Culiacán, México.

*** Doctor en Ciencias (PhD). Facultad de Educación Física de la Universidad Autónoma de Sinaloa. Culiacán, México.

**** Doctor en Ciencias (PhD). Universidad Autónoma de Occidente (UAdeO). Culiacán, México.

***** Doctor en Ciencias de la Actividad Física y el Deporte (PhD). Universidad Autónoma de Sinaloa. Culiacán, México.
} 
Ramírez, K., Valdez-Torres, J. C., Paredes-Osuna, S., Valdez-Lafarga, C., \& Hernández, J. A. (2020). Calidad de vida de pacientes con depresión leve o moderada en México. Revista Ciencias de la Actividad Física UCM, N²1(1), enero-junio, 1-15. DOI: http://doi.org/10.29035/rcaf.21.1.7

\begin{abstract}
Objective. To identify the general characteristics by gender and degree of overweight and/ or obesity and its effect on the quality of life of patients with mild and moderate depression in Sinaloa, Mexico. Material and Methods. A cross-sectional study was conducted with 182 patients with mild to moderate depressive diagnoses. The sample was characterized by gender and degree of overweight and/or obesity, and its impact and association on quality of life were determined. Quality of life was determined with the SF-36 survey. Results. Significant differences were found between patients with mild and moderate depression ( $p=0.000$ ). Physical role was significantly affected by gender ( $p=0.047$ ), while physical function ( $p=0.016$ ) and pain $(p=0.040)$ by the degree of overweight and/or obesity in patients with mild depression. Furthermore, significant correlations were found between physical function and pain with physical role, general health and vitality. Conclusions. Mild depression affected the quality of life dimensions of physical function, pain, and physical role. Depression and the dimensions of physical function and pain are affected by the degree of overweight and/or obesity in patients with mild depression.
\end{abstract}

\title{
Key words
}

Depression, Overweight and Obesity, Quality of life.

\section{INTRODUCCIÓN}

\section{Calidad de vida}

El concepto multidisciplinario de calidad de vida se compone de múltiples y variadas dimensiones, interrelacionadas de manera compleja para conocer la percepción de las personas acerca de su bienestar subjetivo y satisfacción con la vida relacionada a la salud (Urzúa \& Caqueo-Urízar, 2012). Este concepto cuenta con cuatro atributos críticos que incluyen (i) el sentimiento de satisfacción con la vida general, (ii) la capacidad mental para evaluar la vida propia como satisfactoria o no, (iii) un aceptable estado de salud físico, mental, social y emocional determinado por los sujetos y (iv) una evaluación objetiva realizada por una persona sobre condiciones de vida (Pérez \& Salamanca, 2015, Assari, 2016).

La evaluación de la calidad de vida relacionada con la salud es un estándar que ha incrementado su importancia en la determinación del impacto de las enfermedades y la efectividad de intervenciones que van más allá de las medidas epidemiológicas (Poblete-Valderrama, Castillo, Sandoval,

Silva \& García, 2015, Fuentes et al., 2019). Una de las herramientas validadas y utilizadas para esta evaluación es la aplicación de formato corto 36 (SF-36, por sus siglas en inglés), que está conformado en escalas que permiten la evaluación de la calidad de vida en dos componentes, el físico y el emocional (Gu et al., 2019)

\section{Depresión}

La depresión es una enfermedad asociada a síntomas de naturaleza emocional que se considera actualmente como un problema de salud pública por su elevada prevalencia y repercusión sobre la calidad de vida de la población. En México, se estima que la depresión en la población abierta, la padece una de cada cinco personas y de éstas, sólo una de cada diez recibe atención médica (Medina-Mora et al., 2003; Wagner et al., 2012). La Encuesta Nacional de Epidemiologia Psiquiátrica reportó que el 9.1\% de la población encuestada sufrió alguna vez en 
la vida un trastorno afectivo, tal como depresión mayor y menor (Medina-Mora et al., 2003). Por otro lado, la Organización Mundial de la Salud (OMS) considera que la depresión en México está sub-diagnosticada, debido a la presencia de síntomas físicos asociados con la enfermedad, sobre todo de naturaleza dolorosa, mismos que pueden ser más evidentes que los síntomas emocionales (OMS, 2001).

\section{Depresión y Calidad de Vida}

La depresión es el trastorno psiquiátrico más estudiado en relación con la calidad de vida. Sin embargo, no hay consenso en la literatura en cuanto a si existe una verdadera asociación entre depresión y calidad de vida o si ésta se debe a una superposición entre ambos. En primer lugar, depresión y calidad de vida se basan en el modelo teórico de bienestar/satisfacción, lo que podría causar una intersección entre los dos constructos (Berlim \& Fleck, 2007). Empíricamente se ha encontrado que la depresión causa una influencia negativa en la percepción de la calidad de vida, lo cual puede sobreestimar la relación entre ellos, provocando una "falacia afectiva" (Aigner et al., 2006). Por otro lado, algunos instrumentos de calidad de vida incluyen elementos de evaluación psicopatológica, es decir, la presencia de síntomas depresivos, en lugar de elementos específicos de la calidad de vida. Esta situación determina la ocurrencia de correlaciones entre las medidas que pueden ser redundante o falsas, ya que puede ocurrir más por elementos superpuestos que para una asociación de hecho (Sivertsen, Bjørkløf, Engedal, Selbæk y Helvik, 2015).

En la literatura, se encuentran muchos estudios empíricos que demuestran la existencia de un impacto de la depresión sobre la calidad de vida. Los resultados sugieren que el trastorno depresivo afecta todas las dimensiones de la calidad de vida, incluso cuando se controla con otras variables como el grado de sobrepeso y obesidad y la presencia de alguna enfermedad del sujeto (Ruiz, Rojas, Serrano, \& López, 2017, Xu et al., 2018, Rodríguez et al., 2019). En estudios comparativos de las puntuaciones de calidad de vida en sujetos deprimidos o con otras condiciones clínicas, se ha encontrado que sujetos con depresión mayor exhiben mayores niveles de tensión en actividades domésticas, así como irritabilidad social, estrés, limitaciones financieras, funcionamiento ocupacional, peor estado de salud y más días perdidos de trabajo que los sujetos sin la presencia de ella (Johnson, Weissman y Klerman, 1992). En particular, en sujetos bajo condiciones médicas crónicas, la depresión puede conducir a una gran disminución sobre la calidad de vida relacionada con la salud y el funcionamiento social, Ilegando a considerarse mayor que el causado por otras enfermedades físicas. Otro factor relevante es que cuanto mayor sea la intensidad de los síntomas depresivos, peor es la evaluación de la salud en pacientes con alguna condición médica crónica (Ormel et al., 1999; Wells y Sherbourne, 1999).

\section{Propósito del estudio}

La depresión y su relación con la calidad de vida es un aspecto de salud pública poco estudiado en México. En particular, debido a la alta prevalencia de la depresión en México (9.2\%) resulta de interés estudiar su impacto en la calidad de vida de los pacientes que presentan depresión (Berenzon, Lara, Robles \& Medina-Mora, 2013). El propósito general de este estudio fue: primero, estimar las características generales del paciente con depresión leve y moderada; segundo, determinar si la calidad de vida y depresión es influenciada y asociada por género y grado de sobrepeso y obesidad. 
Ramírez, K., Valdez-Torres, J. C., Paredes-Osuna, S., Valdez-Lafarga, C., \& Hernández, J. A. (2020). Calidad de vida de pacientes con depresión leve o moderada en México. Revista Ciencias de la Actividad Física UCM, N²1(1), enero-junio, 1-15. DOI: http://doi.org/10.29035/rcaf.21.1.7

\section{MATERIAL Y MÉTODOS}

\section{Diseño del estudio y muestra}

El estudio es descriptivo-analítico, desarrollado entre 2009 y 2010, con pacientes diagnosticados con depresión leve o moderada. Todos los pacientes fueron diagnosticados por un médico especialista titular en salud mental adscrito en cada una de las sedes: Hospital Psiquiátrico en Culiacán (sede 1), Sinaloa y los Hospitales Generales de Mazatlán (sede 2), Mochis (sede 3), Guasave (sede 4), Guamúchil (sede 5) en el estado de Sinaloa, México. La recolección de los datos fue realizada por investigador adscrito a la sede 1. La muestra consistió de 182 pacientes, seleccionados al azar mensualmente, tomando como características de inclusión: depresión leve o moderada diagnosticada, edad entre 18 a 65 años, emocionalmente apto, no padecer ninguna enfermedad médica agregada y con deseo de participar voluntariamente.

\section{Medición de la Severidad de la depresión}

Para medir la severidad de los síntomas de la depresión se utilizó la escala modificada de Hamilton de 17 preguntas. El rango de puntuación en la escala de 17 preguntas en la versión en español es de 0-54. Una puntuación de 0 a 7 se considera que es normal, de 8 a 17 leve y las puntuaciones de 18 a 24 indican depresión moderada, de acuerdo a la recomendación de la asociación Psiquiátrica Americana (Rush, 2000). La puntuación total, obtenida expresa, además de la gravedad de la depresión, la significancia del cuadro clínico como melancolía, ansiedad y sueño (Bagby, Ryder, Schuller y Marshall, 2004).

\section{Medición de la calidad de vida}

La calidad de vida para la salud se determinó mediante el cuestionario SF-36. El contenido de las preguntas se centra en el estado funcional y el bienestar emocional. Su ámbito de aplicación abarca en población general y pacientes, y se emplea en estudios descriptivos y de evaluación. Este cuestionario detecta estados de salud positivos y negativos y explora la salud física y mental del paciente depresivo; consta de 8 dimensiones, de las cuales 4 corresponden a aspectos físicos (función física, rol físico, dolor corporal y salud general) y las otras 4 a aspectos del área psíquica o mental (vitalidad, función social, rol emocional, salud mental) (Gu et al., 2019). Contiene un total de 36 preguntas y las respuestas tienen diferente valor que depende del número de posibilidades de respuesta para cada pregunta. El rango de puntuación de las respuestas para cada dimensión va desde 0 a 100, que es directamente proporcional al estado de salud; cuanto mayor sea, mejor estado de salud.

\section{Otras variables}

Adicionalmente a las variables de depresión y calidad de vida se registraron; género, edad y grado de obesidad de los sujetos fueron registradas. La edad fue establecida como un criterio de inclusión y exclusión. La obesidad fue clasificada de acuerdo al valor del índice de masa corporal (IMC) y en concordancia con el criterio establecido por la OMS se clasificaron en tres niveles: normopeso con IMC entre 18.5 y 24.99, sobrepeso con IMC entre 25 y 29.99 y obesidad con IMC z 30 (Pérez, \& Salamanca, 2015)

\section{Aspectos éticos}

Todos los sujetos de estudio participaron de forma voluntaria para su inclusión en este estudio mediante la firma de un documento de consentimiento informado por escrito. Este protocolo fue aprobado por los directivos, jefes de enseñanza, médicos psiquiatras, psicólogos y médicos de primer contacto del Hospital Psiquiátrico de Sinaloa y los Hospitales Generales de Mazatlán, 
Mochis, Guasave, Guamúchil en el estado de Sinaloa, México. El estudio fue conducido por médico especialista en salud mental establecido en el Hospital Psiquiátrico de Culiacán.

\section{Análisis estadístico}

El tratamiento de los datos se realizó mediante el paquete estadístico SPSS 22.0. La distribución de la muestra se estableció en valores porcentuales. La estadística descriptiva de los resultados se muestra mediante el valor de la media \pm desviación estándar. El estudio de la normalidad de la distribución de las variables fue examinada con las pruebas de la normalidad de KolmogorovSmirnov y Shapiro-Wilk. La comparación de los valores medios por género (mujeres vs hombres) se empleó prueba t Student para muestras independientes. La comparación de los valores medios por grado de depresión (leve vs moderada) se realizó con la prueba $U$ de Mann-Whitney para muestras independientes. Para la comparación de las medias entre grupos (normopeso, sobrepeso y obesidad) se usó el valor F con la prueba de ANOVA. Cuando se logró una $F$ significativa, se realizaron procedimientos post hoc de Tukey para identificar las diferencias por pares entre las medias. Las relaciones entre las variables de calidad de vida se determinaron mediante el coeficiente de correlación lineal de Pearson. El nivel de la significación se estableció en $p \leq 0.05$.

\section{RESULTADOS}

\section{Características generales de la muestra}

La distribución general de la muestra de los sujetos de este estudio de acuerdo al género, grado de sobrepeso y obesidad y el grado de depresión leve y moderada se muestran en la Tabla 1. La muestra consistió de 182 pacientes, de los cuales, 115 (63.19\%) fueron mujeres [edad media de $39.8 \pm 13.9$ años (IC 95\%, 37.3 - 42.4)] y 67 (36.81\%) hombres [edad media de $30.9 \pm 10.7$ años (IC 95\%, 28.3 - 35.3)]. Considerando el grado de depresión leve y moderada las mujeres presentaron $96.5 \%(n=111)$ y $3.4 \%(n=4)$, mientras que los hombres presentaron $86.4 \%$ ( $n=58$ ) y 13.4 ( $n$ = 9)\%, respectivamente. En la categoría de sobrepeso y obesidad ambos géneros presentaron porcentajes muy similares 63\% (n $=72)$ y $66 \%(n=44)$, para mujeres y hombres, respectivamente (Tabla 1).

Tabla 7

Distribución de la muestra de estudio basado por género y nivel de sobrepeso y obesidad en pacientes con depresión leve y moderada.

\begin{tabular}{rcccccccccccc}
\hline & \multicolumn{1}{c}{ Mujeres } & \multicolumn{4}{c}{ Hombres } \\
\hline Depresión & Leve & \multicolumn{1}{c}{ Moderada } & Leve+Moderada & Leve & Moderada & Leve+Moderada \\
\hline Clasificación IMC & $\mathrm{N}$ & $\%$ & $\mathrm{~N}$ & $\%$ & $\mathrm{~N}$ & $\%$ & $\mathrm{~N}$ & $\%$ & $\mathrm{~N}$ & $\%$ & $\mathrm{~N}$ & $\%$ \\
\hline Normal & 42 & 38 & 1 & 25 & 43 & 37 & 21 & 36 & 2 & 22 & 23 & 34 \\
\hline Sobrepeso & 33 & 30 & 1 & 25 & 34 & 30 & 19 & 33 & 5 & 56 & 24 & 36 \\
\hline Obesidad & 36 & 32 & 2 & 50 & 38 & 33 & 18 & 31 & 2 & 22 & 20 & 30 \\
\hline Grupo & 111 & 100 & 4 & 100 & 115 & 100 & 58 & 100 & 9 & 100 & 67 & 100 \\
\hline
\end{tabular}

La muestra consistió en 182 pacientes, de los cuales 115 (63.19\%) fueron del género femenino y 67 (36.81\%) del masculino. 


\section{Depresión y calidad de vida por género y grado de sobrepeso y obesidad}

El grado de depresión entre hombres (16.1 puntos) y mujeres (16.3 puntos) no se observaron diferenciassignificativas ( $p=.551)$, ambos grupos promediando valore medios de depresión leve. Cuando se comparó la variable depresión por el grado de sobrepeso y obesidad en cada género por separado no se encontraron diferencias significativas. Al contrastar, las variables de calidad de vida, en cada género independientemente, por grado de sobrepeso y obesidad no se observaron diferencias significativas. Sin embargo, al comparar los valores medios de cada variable de calidad de vida, en la variable de rol físico las mujeres (51.2 puntos) presentaron valores significativamente ( $p$ = 0.047) más bajos que los hombres (62.8 puntos). Todos los valores medios entre hombres y mujeres en las otras variables de calidad de vida no presentaron diferencias significativas (Tabla 2).

\section{Depresión y Calidad de vida por grado de sobrepeso y obesidad}

Los niveles de depresión fueron significativamente diferentes entre los grados de sobrepeso y obesidad ( $\mathrm{p}=$ 0.035). Las personas con obesidad ( $n=58$ ) presentaron valores significativamente más bajos que las personas con sobrepeso ( $n=58$ ). Las variables de calidad de vida de función física ( $p=0.049)$ y dolor $(p=0.023)$ presentaron diferencias significativas entre los grados de sobrepeso y obesidad. En la variable de función física el grupo con obesidad presento valores medios más altos ( $p=0.040$ ) que el de sobrepeso. Mientras que en la variable dolor el grupo con obesidad presentaron valores medios más altos que al de normopeso ( $p=0.047$ ). Todas las otras variables de calidad de vida no presentaron diferencias significativas entre el grado de sobrepeso y obesidad. 
Ramírez, K., Valdez-Torres, J. C., Paredes-Osuna, S., Valdez-Lafarga, C., \& Hernández, J. A. (2020). Calidad de vida de pacientes con depresión leve o moderada en México. Revista Ciencias de la Actividad Física UCM, № 21(1), enero-junio, 1-15. DOI: http://doi.org/10.29035/rcaf.21.1.7

Tabla 2

Comparación de la calidad de vida y depresión por género e IMC.

\begin{tabular}{|c|c|c|c|c|c|c|}
\hline \multirow[t]{2}{*}{ Variables } & \multicolumn{2}{|c|}{ Mujeres } & \multicolumn{2}{|c|}{ Hombres } & \multirow[t]{2}{*}{$F$} & \multirow[t]{2}{*}{ Sig. } \\
\hline & $\mathrm{N}$ & Media \pm d.s. & $N$ & Media \pm d.s. & & \\
\hline \multicolumn{7}{|l|}{ Depresión } \\
\hline \multicolumn{7}{|l|}{ Hamilton } \\
\hline Normal & 43 & $16,0 \pm 2,0$ & 23 & $16,2 \pm 2,1$ & & \\
\hline Sobrepeso & 34 & $16,7 \pm 1,6$ & 24 & $16,9 \pm 2,9$ & 2,525 & ,085 \\
\hline Obesidad & 38 & $15,7 \pm 2,3$ & 20 & $15,8 \pm 2,7$ & & \\
\hline Todo grupo & 115 & $16,1 \pm 2,0$ & 67 & $16,3 \pm 2,6$ & 2,651 &, 551 \\
\hline \multirow{2}{*}{\multicolumn{7}{|c|}{$\begin{array}{l}\text { Calidad de vida } \\
\text { Funión Física }\end{array}$}} \\
\hline & & & & & & \\
\hline Normal & 43 & $79,9 \pm 13,0$ & 23 & $79,8 \pm 14,3$ & & \\
\hline Sobrepeso & 34 & $77,8 \pm 12,7$ & 24 & $75,2 \pm 14,3$ &, 840 &, 434 \\
\hline Obesidad & 38 & $81,7 \pm 12,7$ & 20 & $84,3 \pm 10,4$ & & \\
\hline Todo grupo & 115 & $79,9 \pm 12,8$ & 67 & $79,5 \pm 13,5$ & ,350 & ,845 \\
\hline \multicolumn{7}{|l|}{ Rol Físico } \\
\hline Normal & 43 & $54,1 \pm 41,2$ & 23 & $60,4 \pm 38,6$ & & \\
\hline Sobrepeso & 34 & $42,2 \pm 35,0$ & 24 & $68,3 \pm 32,5$ & 1,331 &, 268 \\
\hline Obesidad & 38 & $56,1 \pm 39,1$ & 20 & $58,8 \pm 35,6$ & & \\
\hline Todo grupo & 115 & $51,2 \pm 38,9$ & 67 & $62,8 \pm 35.3^{*}$ & 2,116 &, 047 \\
\hline \multicolumn{7}{|l|}{ Dolor } \\
\hline Normal & 43 & $32,2 \pm 23,4$ & 23 & $32,8 \pm 17,9$ & & \\
\hline Sobrepeso & 34 & $43,7 \pm 20,7$ & 24 & $39,5 \pm 26,9$ & ,788 &, 459 \\
\hline Obesidad & 38 & $43,4 \pm 25,4$ & 20 & $40,8 \pm 21,8$ & & \\
\hline Todo grupo & 115 & $39,3 \pm 23,8$ & 67 & $37,6 \pm 22,6$ &, 501 &, 636 \\
\hline
\end{tabular}


Ramírez, K., Valdez-Torres, J. C., Paredes-Osuna, S., Valdez-Lafarga, C., \& Hernández, J. A. (2020). Calidad de vida de pacientes con depresión leve o moderada en México. Revista Ciencias de la Actividad Física UCM, N²1(1), enero-junio, 1-15. DOI: http://doi.org/10.29035/rcaf.21.1.7

Tabla 3

Comparación de la calidad de vida y depresión por grado de sobrepeso y obesidad.

\begin{tabular}{|c|c|c|c|c|c|c|c|c|}
\hline \multirow[t]{2}{*}{ Variables } & \multicolumn{2}{|c|}{ Normopeso } & \multicolumn{2}{|c|}{ Sobrepeso } & \multicolumn{2}{|r|}{ Obesidad } & \multirow[t]{2}{*}{$F$} & \multirow[t]{2}{*}{ Sig. } \\
\hline & $N$ & Media \pm d.s. & $N$ & Media \pm d.s. & $N$ & Media \pm d.s. & & \\
\hline \multicolumn{9}{|l|}{ Depresión } \\
\hline Hamilton & 66 & $16,1 \pm 2,0$ & 58 & $16,8 \pm 2,2$ & 58 & $15,7 \pm 2.4^{*}$ & 3,41 & ,035 \\
\hline \multicolumn{9}{|l|}{ Calidad de vida } \\
\hline Función Física & 66 & $79,8 \pm 13,3$ & 58 & $76,7 \pm 13,3$ & 58 & $82,6 \pm 11.9^{*}$ & 3,00 &, 050 \\
\hline Rol Físico & 66 & $56,3 \pm 40,1$ & 58 & $53,0 \pm 36,1$ & 58 & $57,0 \pm 37,7$ & ,181 & ,834 \\
\hline Dolor & 66 & $32,4 \pm 21,5$ & 58 & $42,0 \pm 23,4$ & 58 & $42,5 \pm 24.0^{* *}$ & 3,87 &, 023 \\
\hline
\end{tabular}

* nivel de significancia $p \leq 0.05$ del grupo de obesidad al de sobrepeso.

** nivel de significancia $p \leq 0.05$ del grupo de obesidad al de normopeso. 
Ramírez, K., Valdez-Torres, J. C., Paredes-Osuna, S., Valdez-Lafarga, C., \& Hernández, J. A. (2020). Calidad de vida de pacientes con depresión leve o moderada en México. Revista Ciencias de la Actividad Física UCM, № 21(1), enero-junio, 1-15. DOI: http://doi.org/10.29035/rcaf.21.1.7

Tabla 4

Comparación de la calidad de vida y depresión por nivel de depresión leve y moderada y grado de sobrepeso y obesidad.

\begin{tabular}{|c|c|c|c|c|c|c|}
\hline \multirow[t]{2}{*}{ Variables } & \multicolumn{2}{|c|}{ Mujeres } & \multicolumn{2}{|c|}{ Hombres } & \multirow[t]{2}{*}{$F$} & \multirow[t]{2}{*}{ Sig. } \\
\hline & $\mathrm{N}$ & Media \pm d.s. & $N$ & Media \pm d.s. & & \\
\hline \multicolumn{7}{|l|}{ Depresión } \\
\hline \multicolumn{7}{|l|}{ Hamilton } \\
\hline Normal & 63 & $15,8 \pm 1,6$ & 3 & $21,3 \pm 3,1$ & & \\
\hline Sobrepeso & 52 & $16,2 \pm 1,2$ & 6 & $21,7 \pm 2,9$ & 4,316 & ,015 \\
\hline Obesidad & 54 & $15,3 \pm 1.8^{* * *}$ & 4 & $21,3 \pm 3,2$ & & \\
\hline Todo grupo & 169 & $15,8 \pm 1,6$ & 13 & $21,5 \pm 2,8$ & 20,467 &, 000 \\
\hline \multicolumn{7}{|l|}{$\begin{array}{l}\text { Calidad de vida } \\
\text { Funión Física }\end{array}$} \\
\hline Normal & 63 & $79,8 \pm 12,9$ & 3 & $81,7 \pm 23,6$ & & \\
\hline Sobrepeso & 52 & $75,8 \pm 13,1$ & 6 & $85,0 \pm 13,0$ & 4,209 &, 016 \\
\hline Obesidad & 54 & $83,0 \pm 12.2^{* * *}$ & 4 & $77,5 \pm 5,0$ & & \\
\hline Todo grupo & 169 & $79,6 \pm 13,0$ & 13 & $81,9 \pm 13,5$ & ,029 &, 529 \\
\hline \multicolumn{7}{|l|}{ Rol Físico } \\
\hline Normal & 63 & $56,7 \pm 40,2$ & 3 & $46,7 \pm 45,1$ & & \\
\hline Sobrepeso & 52 & $51,5 \pm 36,5$ & 6 & $65,8 \pm 32,6$ & 0,295 &, 745 \\
\hline Obesidad & 54 & $56,1 \pm 38,8$ & 4 & $68,8 \pm 12,5$ & & \\
\hline Todo grupo & 169 & $54,9 \pm 38,5$ & 13 & $62,3 \pm 30,0$ & 3,726 &, 501 \\
\hline \multicolumn{7}{|l|}{ Dolor } \\
\hline Normal & 63 & $32,7 \pm 21.5^{*}$ & 3 & $25,8 \pm 24,0$ & & \\
\hline Sobrepeso & 52 & $42,4 \pm 23,9$ & 6 & $37,9 \pm 19,3$ & 3,289 &, 040 \\
\hline Obesidad & 54 & $41,9 \pm 24.4^{* *}$ & 4 & $50,0 \pm 19,5$ & & \\
\hline Todo grupo & 169 & $38,6 \pm 23,5$ & 13 & $38,8 \pm 20,7$ &, 687 & ,975 \\
\hline
\end{tabular}

* nivel de significancia $p \leq 0.05$ del grupo de normopeso al de sobrepeso.

** nivel de significancia $p \leq 0.05$ del grupo de normopeso al de obesidad.

*** nivel de significancia $\mathrm{p} \leq 0.05$ del grupo de sobrepeso al de obesidad. 
Ramírez, K., Valdez-Torres, J. C., Paredes-Osuna, S., Valdez-Lafarga, C., \& Hernández, J. A. (2020). Calidad de vida de pacientes con depresión leve o moderada en México. Revista Ciencias de la Actividad Física UCM, N²1(1), enero-junio, 1-15. DOI: http://doi.org/10.29035/rcaf.21.1.7

Tabla 5

Nivel de correlación de las dimensiones del SF-36 en pacientes con depresión.

\begin{tabular}{|c|c|c|c|c|}
\hline \multirow[b]{2}{*}{ Físicas } & \multicolumn{2}{|c|}{ Mujeres } & \multicolumn{2}{|c|}{ Hombres } \\
\hline & Función física & Dolor corporal & Función física & Dolor corporal \\
\hline Rol físico & $0.421^{*}$ & -0.130 & $0.362^{*}$ & -0.148 \\
\hline Salud general & -0.074 & $-0.244^{*}$ & 0.044 & $-0.227^{*}$ \\
\hline Vitalidad & -0.003 & - 0.233* & -0.026 & - 0.348* \\
\hline
\end{tabular}

\section{Depresión y calidad de vida por nivel de depresión y grado de sobrepeso y obesidad}

Al comparar la variable depresión entre los grupos de depresión leve al de moderada, el grupo con depresión leve (15.8 puntos) presento valores significativamente más bajo $(p=0.000)$ al grupo con depresión moderada (21.5 puntos). Además, al comparar la variable depresión entre los diferentes grados de sobrepeso y obesidad en el grupo de depresión leve se observaron diferencias significativas ( $P=0.015)$. $E l$ grupo con sobrepeso presento valores significativamente más altos ( $p=0.011$ ) que los obesos. $Y$ al comparar las variables de calidad de vida en los participantes con depresión leve se observaron diferencias significativas entre los grados de sobrepeso y obesidad en las categorías de función física $(p=0.016)$ y dolor $(p=0.040)$. En la variable función física el grupo de sobrepeso presentó valores significativamente más bajos ( $p=0.012$ ) que los obesos, mientras que en la variable dolor el grupo de normopeso presento valores significativamente más bajos al grupo de sobrepeso $(p=0.026)$ y al de obesidad $(p=0.034)$. Las otras variables de calidad de vida no presentaron diferencias significativas entre los grado de sobrepeso y obesidad ni en los valores medios en los grupos con depresión leve y moderada.
En Tabla 5 se muestran las correlaciones entre las dimensiones del SF-36 por género, dónde se destacan de 6 correlaciones compartidas y similares entre mujeres y hombres. En ambos géneros se presento la misma tendencia, la función física presento correlación positiva con el rol físico, el dolor corporal correlaciono negativamente con la salud en general y el dolor corporal correlaciono negativamente con la vitalidad.

\section{DISCUSIÓN}

El presente estudio se realizó para determinar si en pacientes con depresión leve y moderada se ven afectados en la calidad de vida relacionada con la salud de acuerdo al género, el grado de sobrepeso y obesidad en pacientes con depresión leve y moderada. Los resultados de este estudio nos muestran, primero, que las mujeres presentan una mayor incidencia depresiva que los hombres, el nivel de depresión no se ve afectado por el género, pero se observó que el grado de sobrepeso y obesidad influye sobre la depresión. Tres variables de calidad de vida relacionada con la salud física se ven afectadas. El rol físico fue mayor en los hombres que en las mujeres, mientras que la función física y dolor son afectadas por el grado de sobrepeso y obesidad en los participantes con depresión leve. La salud general y las variables de calidad de vida relacionadas con la salud mental no fueron afectadas. 


\section{Depresión}

En este estudio se evaluaron pacientes diagnosticados depresivos con severidad leve y moderada. Los resultados de este estudio presentaron una relación de incidencia mayor en las mujeres depresivas por cada varón deprimido. Estos resultados están en línea con lo observado en otros estudios, donde el género femenino predomina en una proporción mayor (2:1) a los hombres (Belló, Puentes-Rosas, MedinaMora \& Lozano 2005). Por otro lado, en este estudio más del $60 \%$ de la muestra presentó sobrepeso u obesidad para ambos géneros. Se ha demostrado que la depresión y los niveles de sobrepeso y obesidad están directamente relacionadas, debido a que un mayor índice de masa corporal se ha correlacionado positivamente con una mayor frecuencia de depresión (Carpenter, Hasin, Allison, \& Faith, 2000, Rodríguez et al., 2019). En el mismo sentido, Luppino et al. (2010) reportaron que sujetos de 11 a 72 años, con IMC alto, incrementaban en 55\% su riesgo a presentar depresión con el tiempo.

\section{Calidad de vida}

En general, las variables de la salud física relacionadas con la calidad de vida afectadas fueron la función física, el rol físico y el dolor. La variable de salud general y las de la salud mental relacionadas con la calidad de vida no fueron afectadas. En particular, el rol físico relacionado con la calidad de vida esta incluye problemas en el trabajo y/o en actividades diarias. En este estudio, las mujeres presentaron valores inferiores que los hombres, lo que nosindica que las mujeres con depresión leve o moderada pueden ser mayormente afectadas para el desarrollo laboral y de las actividades de la vida diaria. Resultados similares fueron reportados en un estudio previo (Chachamovich, Fleck, Laidlaw \& Power, 2008). Las variables de función física y dolor relacionadas con la calidad de vida fueron afectadas por el IMC en los pacientes con depresión leve (Ver tablas 3 y 4). En este estudio los valores medios de función física fueron más altos en los obesos que en los pacientes con sobrepeso, estos resultados nos indican que no necesariamente a mayores niveles de obesidad menor afectación en la función física en pacientes con depresión leve, lo que nos muestra que existen otras variables asociadas a la depresión y los grados de sobrepeso que no han sido consideradas en el presente estudio. Moderadores como el género, la edad, el origen étnico y el estado socioeconómico afectan las asociaciones de obesidad / depresión a través de las diferencias en la forma en que se experimenta la obesidad en el individuo (Faith, Matz \& Jorge, 2002, Stunkard, Faith, \& Allison, 2003, Wardle \& Cooke, 2005). Estas evidencias nos muestran que aunque el sobrepeso y la obesidad es una variable que interviene en el proceso depresivo del paciente con leve o moderada depresión no es la única variable en el proceso depresivo lo que nos debe llevar a un cuidado con la interpretación de los datos en los procesos de tratamiento y recuperación del paciente.

El dolor ha sido otra variable que fue afectada por los niveles de sobrepeso y obesidad en los pacientes de este estudio. Los resultados de este estudio no muestran que si se considera la variable de dolor independientemente del grado de depresión leve o moderada (Tabla 3) el dolor relacionado con la calidad de vida es menor en los pacientes con normopeso que los obesos. Pero, al considerar solamente al grupo de pacientes con depresión leve (Tabla 4), los pacientes con normopeso presentan menor dolor que los pacientes con sobrepeso y que los obesos. Estas evidencias nos resaltan que aquellos pacientes con depresión leve podrían presentar mayor dolor a mayor grado de sobrepeso y obesidad (Wardle \& Cooke, 2005). En los pacientes con depresión moderada no se observaron diferencias 
Ramírez, K., Valdez-Torres, J. C., Paredes-Osuna, S., Valdez-Lafarga, C., \& Hernández, J. A. (2020). Calidad de vida de pacientes con depresión leve o moderada en México. Revista Ciencias de la Actividad Física UCM, N²1(1), enero-junio, 1-15. DOI: http://doi.org/10.29035/rcaf.21.1.7

significativas en ninguna de las variables de calidad de vida relacionada con la salud, esto puede ser posiblemente a la pequeña muestra analizada.

En el presente estudio, el nivel de depresión no fue relacionado significativamente con la calidad de vida y los niveles de sobrepeso y obesidad de los pacientes. Sin embargo, al considerar las variables afectadas del estado de salud física de este estudio se observó una asociación entre las variables relacionadas con la calidad de vida, observándose un patrón similar tanto para hombres y mujeres. La función física se asoció significativamente con el rol físico, mientras que el dolor corporal fue correlacionado negativamente con salud general y la vitalidad (Ver Tabla 5). Estos resultados de este estudio nos indican, primero, un patrón homogéneo en las correlaciones encontradas entre las variables relacionadas con la calidad de vida; segundo, la estrecha relación entre la funciones del desempeño físico, y por último, los pacientes con depresión leve y moderada que presentan dolor se ve afectada negativamente tanto su salud general como vitalidad. En estudios previos se ha demostrado que la calidad de vida no se modifica al aumentar la edad, o el género en individuos mayores a los 65 años, pero si es influenciada por el estado educativo, la presencia de una enfermedad crónica, y el nivel de depresión e intensidad de dolor afecta negativamente la calidad de vida (Akyol, Durmuş, Doğan, Bek \& Cantürk, 2010). Demura \& Sato (2003) reportaron que la depresión en adultos mayores se relaciona de manera más significativa con el estilo de vida y las actividades sociales. Una de las debilidades de este estudio fue que no se correlacionaron variables demográficas, de estilo de vida, ni sociales con las variables de calidad de vida. En México, un estudio asoció un mayor índice de depresión con una mayor intensidad de dolor, donde el $73.7 \%$ de los pacientes con trastorno depresivo mayor reportaron síntomas físicos dolorosos como dolor muscular, dolor cervical y cefalea con más frecuencia y de mayor intensidad (Ruiz et al., 2007). En este estudio no se encontró una relación entre los índices de depresión con el dolor relacionado con la calidad de vida, esto puede ser posible a que los valores medios de depresión de los sujetos de este estudio fueron bajos lo que nos llevó a no encontrar una asociación significativa entre depresión y dolor. En este estudio no se encontraron diferencias significativas en los pacientes con moderada depresión esto puede ser debido a la relativa pequeña muestra.

\section{CONCLUSIONES}

Este estudio nos confirma que las mujeres presentan mayores cuadros depresivos en relación a los hombres. El 60\% de los pacientes de ambos géneros presentaron sobrepeso y obesidad. Las variables de la salud física relacionadas con la calidad de vida que fueron afectadas fueron la función física y el dolor por el grado de sobrepeso y obesidad en pacientes con depresión leve. No se observaron afectaciones en la salud mental relacionadas con la calidad de vida en pacientes con depresión leve o moderada. En ambos géneros se observó un patrón similar al encontrar relaciones significativas entre las dimensiones función física y dolor con las dimensiones de rol físico, salud general y vitalidad.

\section{Conflicto de intereses}

Los autores declaran que no hay conflictos de interés potenciales con respecto a la investigación, autoría y / o publicación de este artículo. 


\section{REFERENCIAS BIBLIOGRÁFICAS}

Aigner, M., Förster-Streffleur, S., Prause, W., Freidl, M., Weiss, M., \& Bach, M. (2006). What does the WHOQOLBref measure? Social Psychiatry and Psychiatric Epidemiology, 47(1), 81-86. DOI: 10.1007/s00127-005-0997-8

Akyol, Y., Durmuş, D., Doğan, C., Bek, Y., \& Cantürk, F. (2010). Quality of life and level of depressive symptoms in the geriatric population. Archives of Rheumatology, 25(4), 165-173. Recuperado de https:// archivesofrheumatology.org/fulltext/369

Assari, S. (2016). Psychosocial correlates of body mass index in the United States: intersection of race, gender and age. Iranian journal of psychiatry and behavioral sciences, 10(2). DOl: 10.17795/ijpbs-3458

Bagby, R. M., Ryder, A. G., Schuller, D. R., \& Marshall, M. B. (2004). The Hamilton Depression Rating Scale: has the gold standard become a lead weight? American Journal of Psychiatry, 167(12), 2163-2177. DOI: 10.1176/appi. ajp.161.12.2163

Belló, M., Puentes-Rosas, E., Medina-Mora, M. E., \& Lozano, R. (2005). Prevalencia y diagnóstico de depresión en población adulta en México. Salud Pública de México, 47(1), S4-S11. Recuperado de http://saludpublica. $\mathrm{mx} / \mathrm{index}$.php/spm/article/view/4683

Berenzon, S., Lara, M. A., Robles, R., \& Medina-Mora, M. E. (2013). Depresión: estado del conocimiento y la necesidad de políticas públicas y planes de acción en México. Salud Pública de México, 55(1), 74-80. Recuperado de http://www.scielo.org. $\mathrm{m} \times /$ scielo.php?script=sci_arttext\&pid =S0036-36342013000100011
Berlim, M. T., \& Fleck, M. P. (2007). Quality of life and major depression Quality of life impairment in schizophrenia, mood and anxiety disorders (pp. 241252) Dordrecht: Springer. DOI: https:// doi.org/10.1007/978-1-4020-5779-3_12

Carpenter, K. M., Hasin, D. S., Allison, D. B., \& Faith, M. S. (2000). Relationships between obesity and DSM-IV major depressive disorder, suicide ideation, and suicide attempts: results from a general population study. American journal of public health, 90(2), 251257. doi: 10.2105/ajph.90.2.251

Chachamovich, E., Fleck, M., Laidlaw, K., \& Power, M. (2008). Impact of major depression and subsyndromal symptoms on quality of life and attitudes toward aging in an international sample of older adults. The Cerontologist, 48(5), 593-602. DOI: $10.1093 /$ geront/48.5.593

Demura, S., \& Sato, S. (2003). Relationships between depression, lifestyle and quality of life in the community dwelling elderly: a comparison between gender and age groups. Journal of physiological anthropology and applied human science, 22(3), 159-166. DOI: 10.2114/ jpa.22.159

Faith, M. S., Matz, P. E., \& Jorge, M. A. (2002). Obesity-depressionassociationsinthe population. Journal of psychosomatic research, 53(4), 935-942. DOI: 10.1016/ s0022-3999(02)00308-2

Fuentes, F. J. G., Campillo, R. R., Lafourcade, R. S., Fuentes, J. E. G., Ruiz, C. A., Thuillier, B. C., ..., \& Álvarez, C. (2019). Estados de ánimo pre y post competitivos en atletas chilenos universitarios de alto rendimiento. Ciencias de la Actividad Física UCM, 20(1), 1-10. DOI https://doi. org/10.29035/rcaf.20.1.7 
Ramírez, K., Valdez-Torres, J. C., Paredes-Osuna, S., Valdez-Lafarga, C., \& Hernández, J. A. (2020). Calidad de vida de pacientes con depresión leve o moderada en México. Revista Ciencias de la Actividad Física UCM, № 21(1), enero-junio, 1-15. DOI: http://doi.org/10.29035/rcaf.21.1.7

Gu, M., Cheng, Q., Wang, X., Yuan, F., Sam, N. B., Pan, H., ..., \& Ye, D. (2019). The impact of SLE on health-related quality of life assessed with SF36: a systemic review and metaanalysis. Lupus, 28(3), 371-382. DOI: $10.1177 / 0961203319828519$

Johnson, J., Weissman, M. M., \& Klerman, G. L. (1992). Service utilization and social morbidity associated with depressive symptoms in the community. Jama, 267(11), 1478-1483. Recuperado de https://pubmed.ncbi.nlm.nih. gov/1538538/

Luppino, F. S., de Wit, L. M., Bouvy, P. F., Stijnen, T., Cuijpers, P., Penninx, B. W., \& Zitman, F. G. (2010). Overweight, obesity, and depression: a systematic review and meta-analysis of longitudinal studies. Archives of general psychiatry, 67(3), 220-229. DOI: 10.1001/archgen psychiatry.2010.2

Medina-Mora, M. E., Borges, G., Muñoz, C. L., Benjet, C., Jaimes, J. B., Fleiz Bautista, C., ... Rodas, L. C. (2003). Prevalencia de trastornos mentales y uso de servicios: Resultados de la Encuesta Nacional de Epidemiología Psiquiátrica en México. Salud mental, 26(4), 1-16. Recuperado de http://www. revistasaludmental.mx/index.php/ salud_mental/article/view/956

OMS. (2001). Informe sobre la salud en el mundo 2007: Salud mental: nuevos conocimientos, nuevas esperanzas. OMS (Organizacion Mundial de la Salud). Recuperado de https://www. who.int/whr/2001/es/

Ormel, J., Vonkorff, M., Oldehinkel, A., Simon, G., Tiemens, B., \& Üstün, T. (1999). Onset of disability in depressed and non-depressed primary care patients. Psychological medicine, 29(4), 847853. DOI: 10.1017/s0033291799008600
Pérez, C. L., \& Salamanca, R. M. V. (2015). Estilo de afrontamiento, optimismo disposicional, depresión, imagen corporal, imc, y riesgo de tca como predictores de calidad de vida relacionada con la salud. Psychologia: Avances de la Disciplina, 9(2), 35-47. Recuperado de http://www.scielo.org. co/scielo.php?script=sci_arttext\&pid =S1900-23862015000200003

Poblete-Valderrama, F., Castillo, C. M., Sandoval, E. D., Silva, P. V., \& García, M. A. (2015). Depresión, cognición y calidad de vida en adultos mayores activos. Ciencias de la Actividad Física UCM, 16(2), 71-77. Recuperado de http://revistacaf.ucm.cl/article/ view/80

Rodríguez, J. E. P., Badillo, X. V., Florez, D. G. P., Rodríguez, R. P., Gómez, J. C. Q., Sanchez, R. D., \& Solorzano, A. (2019). Depresión y ansiedad y su relación con el perfil antropométrico de los pacientes en rehabilitación cardíaca fases I y |I. CorSalud, 77(4), 287-295. Recuperado de http://www. revcorsalud.sld.cu/index.php/cors/ article/view/415

Ruiz Acacio, E. A., Rojas Russell, M. E., Serrano Alvarado, K., \& López Cervantes, M. (2017). Relación entre el sobrepeso u obesidad y la calidad de vida relacionada con la salud: el rol mediadory moderador de la ansiedad y la depresión. Revista Psicología y Salud, 27(2). Recuperado de http:// psicologiaysalud.uv.mx/index.php/ psicysalud/article/view/2533 
Ruiz Flores, L. G., Colín Piana, R. F., Corlay Noriega, I. S.-Y., Muñoz, L., del Carmen, M., \& Dueñas Tentori, H. J. (2007). Trastorno depresivo mayor en México: la relación entre la intensidad de la depresión, los síntomas físicos dolorosos y la calidad de vida. Salud mental, 30(2), 25-32. Recuperado de http://www.revistasaludmental. $\mathrm{mx} / \mathrm{index}$.php/salud_mental/article/ view/1155

Rush, J. (2000). Handbook of Psychiatric Measures. Washington, DC : American Psychiatric Association, pp. 166-168.

Sivertsen, H., Bjørkløf, G. H., Engedal, K., Selbæk, G., \& Helvik, A. S. (2015). Depression and quality of life in older persons: a review. Dementia and geriatric cognitive disorders, 40(5-6), 311-339. DOI: 10.1159/000437299

Stunkard, A. J., Faith, M. S., \& Allison, K. C. (2003). Depression and obesity. Biological psychiatry, 54(3), 330-337. DOI: 10.1016/s0006-3223(03)00608-5

Urzúa, A., \& Caqueo-Urízar, A. (2012). Calidad de vida: Una revisión teórica del concepto. Terapia psicológica, 30(1), 61-71. DOI: http://dx.doi.org/10.4067/ S0718-48082012000100006

Wagner, F. A., González-Forteza, C., SánchezGarcía, S., García-Peña, C., \& Gallo, J. J. (2012). Enfocando la depresión como problema de salud pública en México. Salud mental, 35(1), 3-11. Recuperado de http://www.scielo.org.mx/scielo. php?script=sci_arttext\&pid=S0185$33252012000100002 \& \mid n g=e s \&$ llng=es

Wardle, J., \& Cooke, L. (2005). The impact of obesity on psychological wellbeing. Best practice \& research clinical endocrinology \& metabolism, 19(3), 421-440. DOI: 10.1016/j. beem.2005.04.006
Wells, K. B., \& Sherbourne, C. D. (1999). Functioning and utility for current health of patients with depression or chronic medical conditions in managed, primary care practices. Archives of general psychiatry, 56(10), 897-904. DOI: https://doi.org/10.1001/ archpsyc.56.10.897

Xu, Y., Guo, S. L., Ma, Y. F., Xu, N., Wu, W. N., Yu, J. C., ..., \& Ye, X. (2018). Relationship between Depression and Quality of Life in Patients with Severe Obesity. Zhongguo yi xue ke xue yuan xue bao. Acta Academiae Medicinae Sinicae, 40(5), 625-629. Recuperado de $\quad$ https://pubmed.ncbi.nlm.nih. gov/30404693/

\section{Dirección para correspondencia}

José Aldo Hernández Murúa

Doctor en Ciencias de la Actividad Física y el Deporte (PhD).

Universidad Autónoma de Sinaloa

Culiacán, México

Dirección postal: Av. de las Américas y Prol.

Josefa Ortíz de Domínguez s/n.

Circuito interior sur-oriente. Ciudad

Universitaria, C.P. 80040.

Reserach ID:

https://orcid.org/0000-0002-9277-5799

Contacto:

aldohdez80@hotmail.com

Recibido: 15-12-2019

Aceptado: 04-06-2020 